Collection SFN 9 (2008) 149-158

(C) EDP Sciences, Les Ulis

DOI: $10.1051 / \mathrm{sfn}: 2008011$

\title{
Ordres complexes et apériodicités
}

\author{
D. Grebille
}

Institut Laboratoire CRISMAT (UMR CNRS 6508), ENSICAEN, Boulevard Maréchal Juin, 14050 Caen Cedex, France

\begin{abstract}
Some basic theoretical considerations showed that the classical description of the crystalline state through a three dimensional periodicity is not sufficient to take into account all the possible long range order atomic structures. This long range order can be associated to a supercrystal described in a superspace of dimension $n>3$. The real aperiodic structure and its experimental diffraction pattern can be recovered through a section-projection method in the superspace. Some actual examples of this aperiodic order are the quasicrystals, the modulated structures and the misfit compounds. Their specificities are described here et some structural examples are given to illustrate the previous theoretical considerations.
\end{abstract}

Résumé. Quelques considérations théoriques simples ont montré que la description de l'ordre cristallin par une triple périodicité n'est pas suffisante pour rendre compte de la notion d'ordre à grande distance. Celle-ci peut être retrouvée à travers une méthode de section-projection d'un supercristal dans un espace de dimension $n>3$. Des réalisations concrètes de cet ordre apériodique sont les quasicristaux, les structures modulées et les structures composites. Leurs particularités sont décrites ici et quelques exemples de structures illustrent les considérations théoriques précédentes.

\section{VOYAGE EN SUPERESPACE}

Très longtemps, les notions d'ordre à longue distance et de triple périodicité ont été systématiquement, implicitement et arbitrairement associées et le cristal a été défini par le triple empilement d'une maille élémentaire définie par 3 vecteurs de base linéairement indépendants $\mathbf{a}$, b et $\mathbf{c}$. Si l'on admet qu'un diagramme de diffraction, transformée de Fourier de la fonction d'autocorrélation de l'échantillon étudié, ne présentant que des signaux ponctuels, traduit intrinsèquement un ordre à grande distance, caractéristique de l'ordre cristallin $[1,2]$, alors l'observation récente de figures de diffraction particulières (Fig. 1) a clairement démontré que la triple périodicité en était curieusement absente (axes de symétrie incompatibles (fig. 1a), réflexions satellites en position incommensurables (Fig. 1b), présence intrinsèque de 2 réseaux partiellement indépendants (Fig. 1c)). Or, un point commun de ces diagrammes est qu'il est possible de les indexer entièrement à l'aide de $n$ vecteurs $\mathbf{a}_{\mathbf{i}}^{*}$, rationnellement indépendants, avec $n>3$. Exploitons ce résultat pour prévoir théoriquement les types d'organisation structurales sous-jacentes à ces observations.

\subsection{Les principes du formalisme de superespace}

Le cristal tripériodique présente une fonction densité qui peut s'exprimer comme la transformée de Fourier de l'image diffractée, à savoir :

$$
\rho(\mathbf{r})=\frac{1}{V} \sum_{h, k, l} F_{h, k, l} e^{-2 \pi i\left(\mathbf{G}_{h, k, l} \bullet \mathbf{r}\right)}
$$

avec $\mathbf{G}_{h, k, l}=h \mathbf{a}^{*}+k \mathbf{b}^{*}+l \mathbf{c}^{*}$, vecteur du réseau réciproque, et $F_{h, k, l}$, facteur de structure correspondant. On peut généraliser très facilement cette expression au cas où l'on observe $n$ vecteurs de 


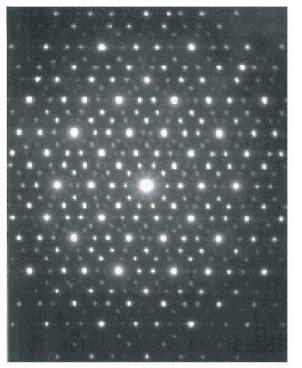

(a)

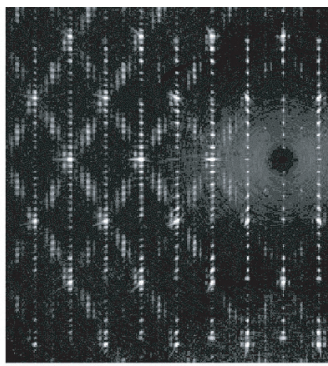

(b)

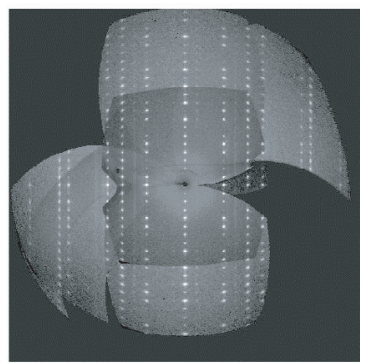

(c)

Figure 1. Exemples de clichés de diffraction de structures apériodiques (a) quasicristal Al-Mn; (b) structure modulée $\left(\mathrm{PO}_{2}\right)_{4}\left(\mathrm{WO}_{3}\right)_{16} ;$ (c) structure composite $\left[\mathrm{Ca}_{2} \mathrm{CoO}_{3}\right]\left[\mathrm{CoO}_{2}\right]_{1.62}$.

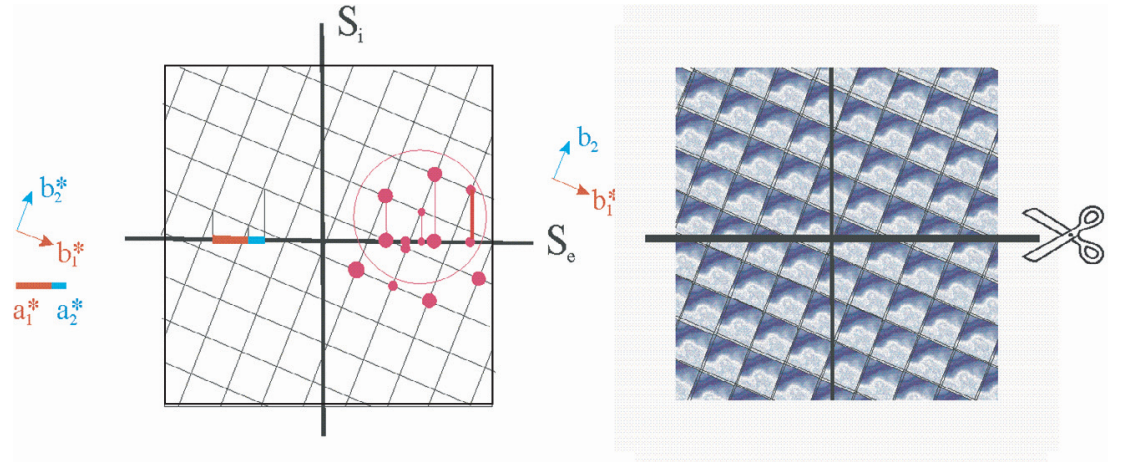

Figure 2. Méthode de projection du réseau réciproque et de section du réseau direct.

base rationnellement indépendants $\mathbf{a}_{i}^{*}, i=1$ à $n$ :

$$
\rho(\mathbf{r})=\frac{1}{V} \sum_{h_{1}, h_{2} \ldots h_{n}} F_{h_{1}, h_{2} \ldots h} e^{-2 \pi i\left(\mathbf{G}_{h_{1}, h_{2} \ldots h_{n}} \bullet \mathbf{r}\right)}
$$

avec $\mathbf{G}_{h_{1}, h_{2} \ldots h_{n}}=\sum_{1}^{n} h_{i} \mathbf{a}_{i}^{*}$ et $F_{h_{1}, h_{2} \ldots h_{n}}$, le facteur de structure de la tache de diffraction observée.

Il est maintenant possible de considérer que les $n$ vecteurs de base $\mathbf{a}_{\mathrm{i}}^{*}$ sont en fait les projections sur l'espace réciproque tridimensionnel (espace externe $\mathrm{S}_{\mathrm{e}}$ ) de $n$ vecteurs de base $\mathbf{b}_{\mathrm{i}}^{*}$, linéairement indépendants dans un «superespace» réciproque de dimension $n$ (Fig. 2a). Le sous-espace orthogonal à l'espace externe $S_{e}$ est l'espace interne $S_{i}$. On peut alors définir une densité généralisée dans le superespace direct correspondant:

$$
\tilde{\rho}(\tilde{\mathbf{r}})=\frac{1}{\tilde{V}} \sum_{h_{1}, h_{2} \ldots h_{n}} F_{h_{1}, h_{2} \ldots h} e^{-2 \pi i\left(\tilde{\mathbf{G}}_{h_{1}, h_{2} \ldots h_{n}} \bullet \tilde{\mathbf{r}}\right)} .
$$

Cette densité caractérise maintenant un «supercristal » avec une périodicité de dimension $n$. D'après les propriétés de la transformée de Fourier, si le réseau réciproque $\mathbf{G}_{h_{1}, h_{2} \ldots h_{n}}$ dans l'espace physique est la projection d'un réseau réciproque $\tilde{\mathbf{G}}_{h_{1}, h_{2} \ldots h_{n}}$ dans le superespace, le cristal réel dans l'espace physique est maintenant la section du supercristal $\tilde{\rho}(\tilde{\mathbf{r}})$ (Fig. 2b). Ainsi, la section du réseau périodique de dimension $n$ caractérisant le supercristal par le sous-espace de dimension 3 correspondant à l'espace externe, n'est plus, en général, un réseau tripériodique, d'où la perte de périodicité observée dans l'espace physique 
et la justification du terme «apériodique». Il est clair que toutes les notions de symétrie cristalline connues dans l'espace de dimension 3 peuvent se généraliser dans le superespace, avec l'introduction éventuelle d'éléments de symétrie incompatibles avec la triple périodicité. La prise en compte de cette «supersymétrie » est d'un intérêt majeur (comme la symétrie 3D pour un cristal 3D) pour la résolution d'une structure, mais sa description dépasse le cadre de cet article. On pourra par exemple se reporter aux Tables Internationales de Cristallographie [3].

\subsection{3 réalisations possibles de structures apériodiques}

Il convient à présent de chercher à décorer la supermaille du nouveau cristal par des objets qui seront la généralisation des atomes d'une structure cristalline classique. Il est raisonnable de chercher à décrire, en première approximation, la structure apériodique par des sites atomiques caractérisés par des points dans l'espace physique, définis par leurs 3 coordonnées d'espace dans un repère donné. Sachant que le cristal est la section du supercristal, de simples points dans le supercristal ne permettraient probablement pas d'atteindre les densités atomiques attendues ; cela induit nécessairement que les objets représentant les atomes dans le supercristal soient représentés par des variétés de dimension $(n-3)$ dans l'espace interne, de telle manière qu'une section de ces objets par l'espace physique se limite à un point dans l'espace externe. Ces variétés peuvent être bornées ou infinies. Dans ce dernier cas, puisqu'elles obéissent à une périodicité dans le superespace, celle-ci induit une périodicité moyenne associée dans l'espace physique.

Considérons dans un premier temps, une structure apériodique de dimension 1, résultant de la section d'un supercristal de dimension 2 [4]. Les atomes seront représentés soit par des courbes bornées (éventuellement des segments de droite), soit par des courbes infinies périodiques. Le premier cas est illustré sur la figure 3a et correspond au cas du quasicristal. On constate que si la section du supercristal se fait selon une orientation irrationnelle quelconque par rapport aux vecteurs de base du supercristal, on perd la périodicité dans l'espace physique et la structure peut se décrire par une alternance apériodique de distances longues et courtes [5]. On conçoit bien ici que l'on garde une structure ordonnée à grande distance sans périodicité réelle. L'exemple type de cet agencement est la séquence de Fibonacci à une dimension, ou les diagrammes de Penrose à deux dimensions. L'espace physique est alors entièrement pavé de façon non périodique par deux «volumes » différents.

Si maintenant on décore le supercristal de dimension 2 par des cordes ondulées (Fig. 3b) respectant bien évidemment la périodicité de dimension 2 du supercristal, la structure réelle dans l'espace physique peut se décrire comme une structure en moyenne périodique (cas où les courbes se réduisent à des droites) mais perturbée par des déplacements atomiques périodiques, dont la période est incommensurable avec la période moyenne. Ceci correspond en d'autres termes à un phonon de fréquence nulle. Nous avons ici le cas de la structure modulée displacive (on pourrait aussi moduler l'occupation des sites).

Enfin, troisième et dernier exemple, où l'on superpose deux jeux de cordes indépendants. Chaque jeu définit en moyenne sa périodicité de base dans la structure réelle, mais on observe maintenant la juxtaposition de deux périodicités indépendantes, soit encore la coexistence dans le cristal apériodique de deux sous-systèmes périodiques. C'est le cas du cristal composite (misfit en anglais) (Fig. 3c). Bien évidemment, chaque sous-système va perturber l'autre sous-système selon sa propre périodicité, et l'on retrouve dans ce cas deux structures modulées imbriquées l'une dans l'autre. Bien évidemment, sur la figure unidimensionnelle $3 \mathrm{c}$, on observe des distances interatomiques qui deviennent localement trop courtes. On peut donc davantage imaginer ce type de structure dans un système 2D ou 3D, permettant, dans les autres dimensions partagées par les deux sous-systèmes, d'accommoder de bonnes distances interatomiques. L'affinement structural de telles structures montre souvent que les modulations réciproques des deux jeux d'atomes permettent d'éviter les distances trop courtes qui seraient induites par des cordes rectilignes [6]. 


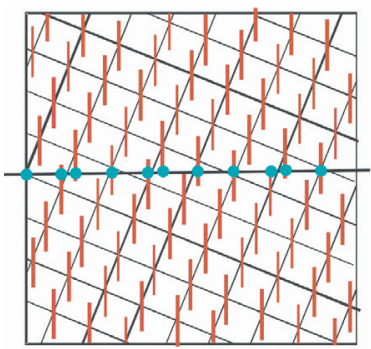

(a)

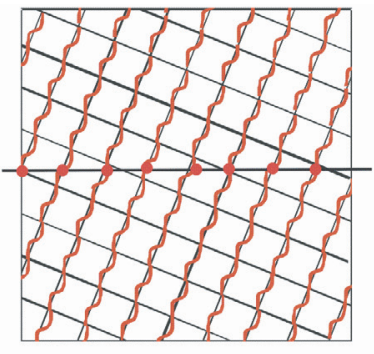

(b)

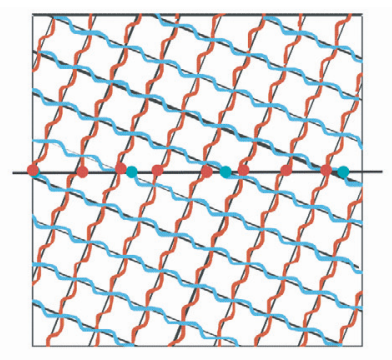

(c)

Figure 3. 3 décorations du supercristal : (a) quasicristal ; (b) structure modulée ; (c) structure composite [4].

Si l'on revient aux systèmes réels déjà observés de structures apériodiques à 3 dimensions, le supercristal pourra se décrire soit à 4 dimensions, avec des atomes sous formes de segments ou de cordes, soit à 5 dimensions, avec des atomes sous formes de surfaces bornées ou de nappes, soit à 6 dimensions, avec des atomes sous formes de volumes délimités par des surfaces atomiques connexes.

Il est alors possible de calculer le diagramme de diffraction du supercristal dans le superespace et de reconstituer ensuite, par projection sur l'espace physique réciproque, le diagramme de diffraction réellement observable par les techniques de diffraction habituelles. Si le réseau réciproque du supercristal était caractérisé par des intensités réparties de manière homogène, la projection d'un tel réseau conduirait à un ensemble dense de taches de diffraction intenses. Il se trouve que compte tenu de la décoration spécifique du supercristal par les cordes, surfaces ou volumes atomiques, les fortes intensités sont regroupées selon des bandes. La projection de ces bandes fait apparaître alors un jeu de taches de diffraction ponctuelles et bien résolues.

Dans le cas du quasicristal (Fig. 4a), aucune réseau tripériodique n’est apparent. Par contre, selon les projections, on peut mettre en évidence des opérations de symétrie du supercristal, qui restent compatibles avec l'opération de projection, et font donc apparaître des symétries a priori incompatibles avec un réseau tridimensionnel, d'où l'aspect spectaculaire et révolutionnaire du cliché de la fig. 1a. Dans le cas de la structure modulée (Fig. 4b), les taches les plus intenses sont réparties de manière périodique. Elles permettent de définir une structure moyenne qui est une structure cristalline classique à 3 dimensions. Par contre, on observe de part et d'autre des taches principales, des réflexions satellites à $\pm n \mathbf{q}^{*}$, qui peuvent être associées, en première approximation aux différents ordres dans

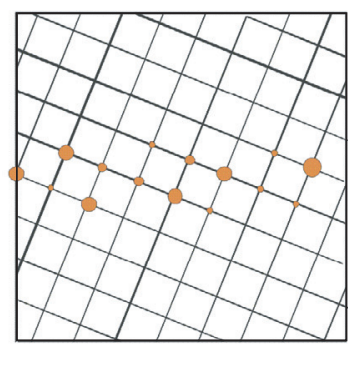

(a)
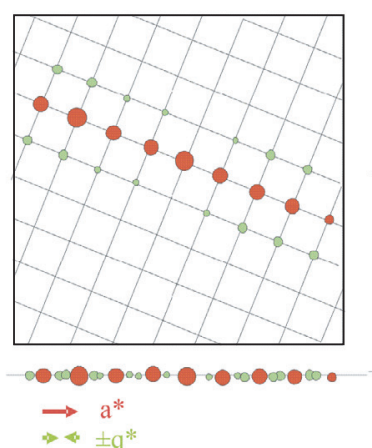

(b)

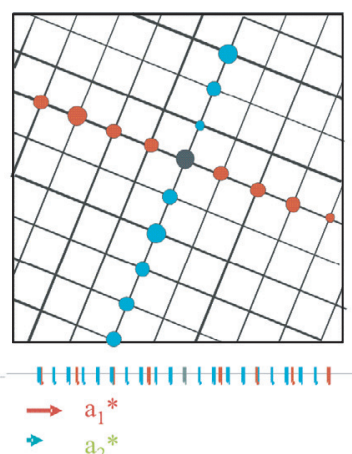

(c)

Figure 4. Diagrammes de diffraction correspondant aux 3 modèles de la figure 3 [4]. 
la décomposition en série de Fourier des fonctions de modulation. Pour le cristal composite (Fig. 4c), on va retrouver deux jeux indépendants de taches principales, caractérisés chacun par son jeu de paramètres réciproques $\mathbf{a}_{1 \mathrm{i}}^{*}$ et $\mathbf{a}_{2 \mathrm{i}}^{*},(i=1$ à 3 ), ainsi que des réflexions satellites, qui peuvent être considérées comme des satellites de l'un ou de l'autre réseau. L'un ou l'autre des deux réseaux peut servir de référence, et on définit pour chaque réseau, une matrice permettant d'associer les $2 \times 3$ vecteurs de base $\mathbf{a}_{1 \mathrm{i}}$ et $\mathbf{a}_{2 \mathrm{i}}$, aux vecteurs de base globaux $\mathbf{a}_{\mathrm{j}}(j=1$ à $\mathrm{n})$ du supercristal. Pour des raisons de clarté, les réflexions satellites sont omises sur la fig. 4c et les taches de diffraction du cristal réel sont représentées par des barres verticales.

\section{DE LA THÉORIE À L'OBSERVATION ET À L'EXPÉRIENCE}

À partir du moment où l'on peut quantifier les intensités diffractées mesurées par les techniques habituelles sur monocristal ou sur poudre, il est possible de généraliser les résolutions structurales dans le superespace, en tenant compte de manière spécifique de la forme des nouvelles entités représentant les atomes d'une part, et des nouvelles notions de symétrie associées au supercristal. Il convient systématiquement après un tel affinement, de décrire ensuite le cristal apériodique réel en opérant la section du supercristal.

\subsection{Le quasicristal}

Les premiers modèles de surfaces atomiques utilisées dans le cadre de la description de quasicristaux étaient simplement des sphères concentriques associées aux espèces atomiques présentes. Une analyse plus rigoureuse et développée a permis de décrire les surfaces atomiques en tenant compte de la symétrie du quasicristal. A partir de motifs élémentaires et en appliquant les différentes opérations de symétrie, il est ainsi possible de construire des surfaces atomiques complexes rendant mieux compte de la réalité expérimentale. Ainsi, dans le cas de l'alliage icosaédrique Al-Pd-Mn [7], un modèle d'amas a pu être proposé sur la base des surfaces atomiques de la figure 5. La structure réelle qui est décrite à partir de ce modèle est obtenue par des sections 3D de tels entités et permet de préciser, dans l'espace physique, les différents types d'amas que l'on peut reconnaître et qui permettent de paver le volume 3D selon le schéma apériodique correspondant.

Les outils habituels d'aide à la résolution structurale ou de représentation des densités électroniques ou nucléaires peuvent aussi être généralisés. On peut donc utiliser les fonctions de Patterson correspondantes, représenter des cartes de Fourier de différents types, et selon différentes orientations, incluant éventuellement les dimensions internes. C'est ainsi que des cartographies obtenues par des

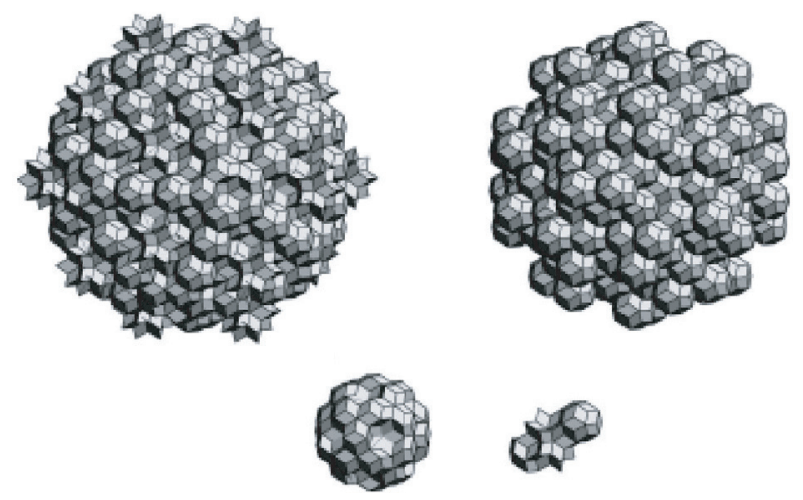

Figure 5. Surfaces atomiques ou domaines d'occupation pour l'alliage icosaédrique Al-Pd-Mn [7]. 


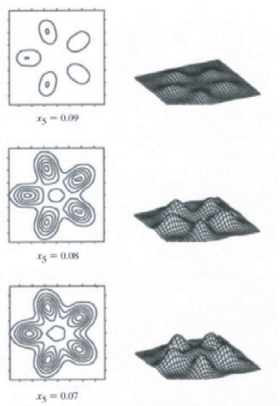

(a)

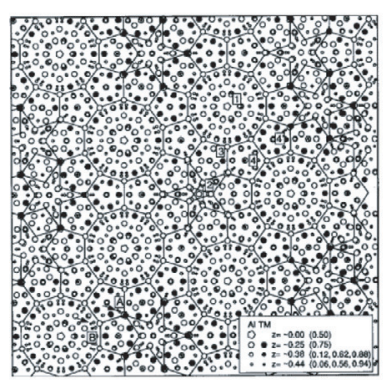

(b)

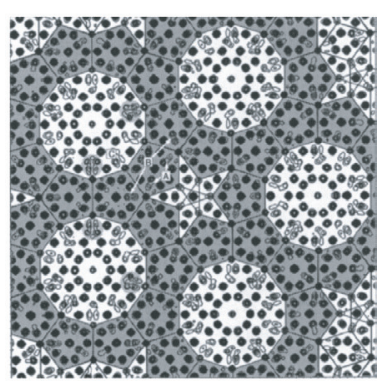

(c)

Figure 6. AlMnPd décagonal : (a) cartographies par maximum d'entropie des surfaces atomiques dans l'espace interne ; (b) cartographie par maximum d'entropie de l'espace externe et (c) projection schématique de la structure affinée [8].

méthodes de maximum d'entropie ont permis, dans le cas de l'alliage décagonal Al-Mn-Pd [8], de reconstruire des sections des surfaces atomiques, présentant en particulier une symétrie 5 (Fig. 6a), ou de reconstruire la densité électronique dans la section correspondant à l'espace physique (Fig. 6b). La structure atomique proposée (Fig. 6c) propose un agencement apériodique d'amas qui correspond particulièrement bien à la reconstruction précédente.

Pour ce qui concerne les techniques neutroniques, la diffusion inélastique est une sonde privilégiée pour caractériser les modes de phonons dans une structure cristalline. Dans le cas des quasicristaux, cette technique a pu également être mise en œuvre. En particulier, elle a été exploitée pour la caractérisation d'un mode spécifique des structures apériodiques, à savoir le phason, lié à une distorsion du réseau dans ses dimensions internes. Dans le cas du quasicristal, on conçoit qu' une telle distorsion puisse avoir pour conséquence le déplacement d'un segment ou d'une surface atomique, de telle sorte que le schéma de section par l'espace physique en soit modifié : on parle dans ce cas de saut atomique.

\subsection{La structure modulée}

Les exemples de structures modulées sont maintenant très nombreux et concernent différents types de structure. Nous nous contenterons ici de donner des exemples d'oxydes de manganèse, qui ont été en particulier étudiés pour leurs propriétés de magnétorésistance, liées à la valence mixte du manganèse, elle-même due à une substitution cationique entre espèce di- et trivalente, par exemple Ca et Pr. Ces structures sont souvent sujettes à des mises en ordre nucléaires, orbitalaires ou magnétiques à basse température.

Dans le cas de la structure $\operatorname{Pr}_{0,5} \mathrm{Ca}_{1,5} \mathrm{MnO}_{4}$ [9], on observe au voisinage de la température ambiante, une transition de phases se traduisant par l'apparition de réflexions satellites en position incommensurable. L'analyse de la structure révèle une structure monoincommensurable, avec un rapport d'incommensurabilité de 0,412. Les échantillons observés présentent le plus souvent un partage en domaines maclés avec un schéma de diffraction en croix de satellites autour de chaque réflexion principale, correspondant à une réflexion de la structure haute température (Fig. 7a). La résolution structurale a pu être menée en tenant compte de ce partage en domaines et a pu mettre en évidence des modulations displacives concernant principalement les oxygènes équatoriaux des octaèdres $\mathrm{MnO}_{6}$ (Fig. 7b). La figure 7b représente une carte de Fourier permettant de bien mettre en évidence la corde atomique ondulée de cet atome d'oxygène. La courbe à l'intérieur de cette corde représente la fonction de modulation affinée par le programme de résolution structurale JANA2000 [10]. 

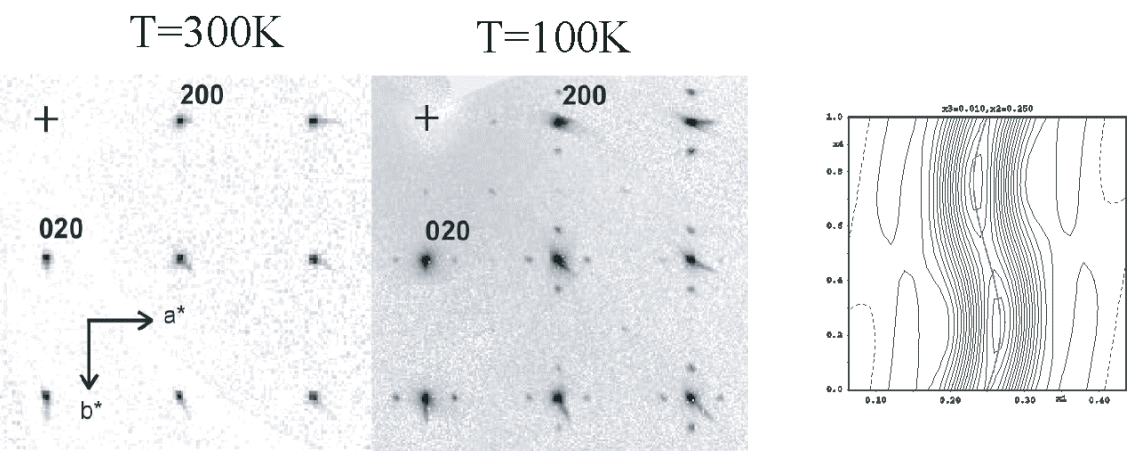

Figure 7. $\mathrm{Pr}_{0,5} \mathrm{Ca}_{1,5} \mathrm{MnO}_{4}$ : diagrammes de diffraction à 300 et à $100 \mathrm{~K}$; fonction de modulation de l'oxygène [9].

Le même type d'étude a pu être mené sur des pérovskites tridimensionnelles telles $\operatorname{Pr}_{0.7} \mathrm{Ca}_{0.3} \mathrm{MnO}_{3}$. Dans ce cas, on conserve à basse température l'apparition de réflexions satellites en position commensurable $(0,5)$, conduisant en fait à une surstructure d'ordre 2 . On peut malgré tout, et en considérant les spécificités de calcul de facteur de structure liées à ce caractère commensurable, traiter cette structure comme une structure modulée. Cela permet de prendre en compte des symétries spécifiques liées à la transition de phases. Si l'on omet ces symétries, on peut se heurter à des corrélations importantes dans la description en termes de surstructure, car on considère comme indépendants des atomes, qui, en fait, restent liés par la symétrie de la structure moyenne. Par ailleurs, on peut montrer que dans ce cas, le choix de la section dans le supercristal pour définir la structure réelle, a son importance et peut conduire à des structures de symétries différentes. Ainsi, dans le cas qui nous intéresse, les deux symétries en question étaient $\mathrm{P} 2_{1} / \mathrm{m}$ ou $\mathrm{P} 2_{1} \mathrm{~nm}$. L'affinement dans le superespace permet alors de comparer les deux solutions dans un même cadre. Compte tenu de la difficulté de ce type d'affinement, nous avons essayé de tirer parti de différentes techniques, à savoir la diffraction de neutrons sur poudre, la diffraction de rayons X sur monocristal monodomaine et la diffraction de neutrons sur monocristal maclé (6 domaines). Les résultats sont schématiquement représentés sur la figure 8 et montrent un cohérence globale satisfaisante. On peut critiquer le résultat obtenu sur poudre, qui amplifie les amplitudes de modulation de l'oxygène, mais qui sont liés au fait que le diagramme expérimental ne présente pratiquement que 4 réflexions satellites véritablement significatives. Les deux autres affinements restent très proches et nous ont permis de trancher pour la solution orthorhombique. Celle-ci est davantage en accord avec un modèle polaronique sans véritable ordre des charges [11] contre un modèle orbitalaire qui avait été introduit pour rendre compte d'une mise en ordre des charges [12].

Une illustration de structure modulée dans l'espace réel peut être fournie par la phase $\mathrm{Bi}_{2} \mathrm{Sr}_{2} \mathrm{CoO}_{6}$ [13], caractérisée par un vecteur de modulation $(0,0.24,1)$. L'affinement se traduit principalement par des modulations displacives transversales selon la direction c d'empilement des couches et longitudinales, principalement dans les couches $\mathrm{BiO}$ de cette structure (Fig. 9). L'onde transversale associée à toutes les opérations de symétrie 4D compatibles avec cette structure, se traduit visuellement par une forte ondulation des feuillets $\mathrm{BiO}-\mathrm{SrO}-\mathrm{CoO}_{2}-\mathrm{SrO}-\mathrm{BiO}$, en opposition de phases selonc. L'onde longitudinale se traduit par une succession selon la direction de modulation, de domaines en compression et en extension, corrélés aux ondulations transverses des feuillets. Dans les domaines en extension, un oxygène supplémentaire peut ainsi trouver sa place, ce qui se traduit globalement par deux réseaux moyens différents dans les couches $\mathrm{BiO}$, un réseau $\mathrm{Bi}$ et un réseau $\mathrm{O}$. Dans ces conditions, cette structure modulée relève en partie également d'une structure composite (voir aussi pour le même type de problème [14-16]). Ceci démontre que les complexités peuvent être doubles et qu'un modèle est parfois insuffisant. 

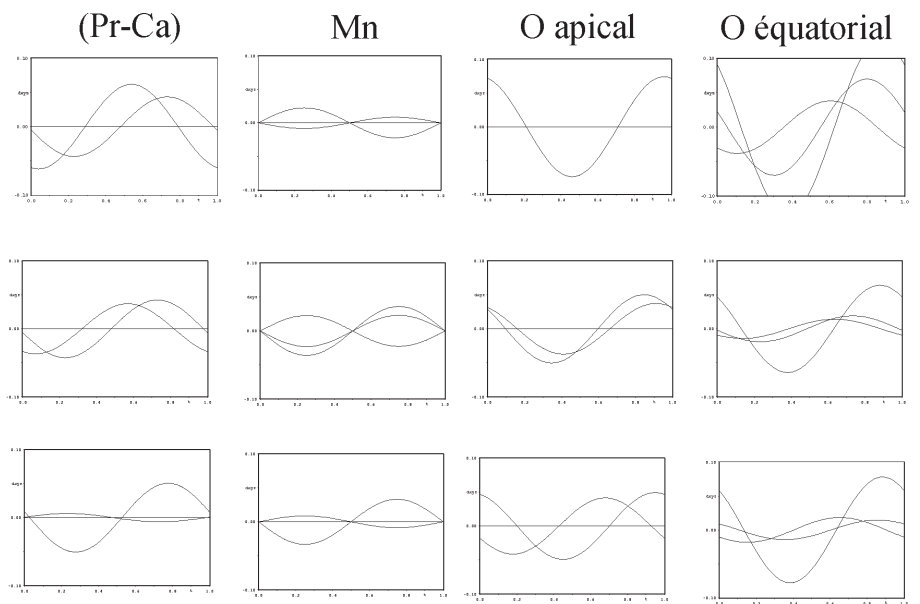

(C)

Figure 8. $\mathrm{Pr}_{0,7} \mathrm{Ca}_{0,3} \mathrm{MnO}_{3}$ : fonctions de modulation de déplacement des différents atomes dans las affinements à partir des données de diffraction (A) de neutrons sur poudre ; (B) de rayons X sur monocristal monodomaine ; (C) de neutrons sur monocristal maclé.

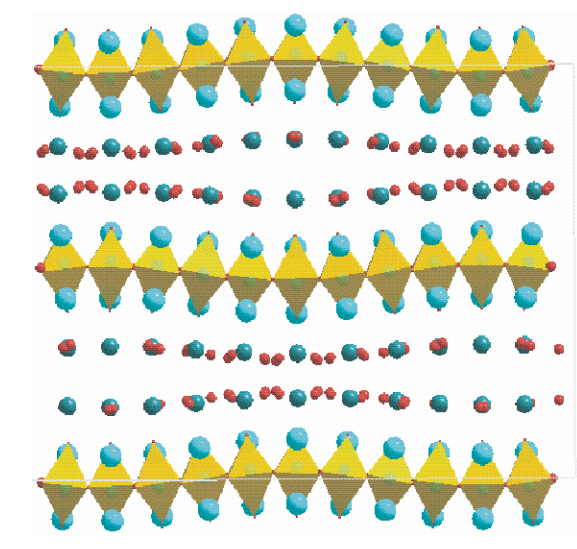

Figure 9. $\mathrm{Bi}_{2} \mathrm{Sr}_{2} \mathrm{CoO}_{6}$ : représentation schématique de la structure.

\subsection{La structure composite}

Pour ce qui concerne les structures composites, nous prendrons l'exemple de l'oxyde de cobalt lamellaire thermoélectrique $\left[\mathrm{Ca}_{2} \mathrm{CoO}_{3}\right]\left[\mathrm{CoO}_{2}\right]_{1.62}$. Le pouvoir thermoélectrique important de ce type de composés est ici lié au dopage en trous de la couche $\mathrm{CoO}_{2}$ qui résulte directement de la non stoechiométrie intrinsèque liée au caractère composite de la structure (deux paramètres a dans un rapport 1,62) (Fig. 10). Des études structurales en diffraction des rayons X sur monocristal ont permis de montrer l'existence d'un désordre cationique et anionique dans la couche centrale du réseau $\left[\mathrm{Ca}_{2} \mathrm{CoO}_{3}\right]$. Pour valider le désordre des atomes d'oxygène de cette couche, moins diffusants pour les rayons $\mathrm{X}$ que les cations environnants, nous avons pu procéder à une résolution structurale à partir d'un diagramme de diffraction des neutrons sur poudre (Fig. 10b). L'affinement ainsi obtenu est tout à fait compatible avec l'affinement précédent et a permis de conforter l'éclatement des atomes d'oxygène [17]. Les figures $10 \mathrm{c}, 10 \mathrm{~d}$ et $10 \mathrm{e}$ montrent bien la spécificité d'une telle structure composite, en particulier sur un diagramme de diffraction sur poudre. Des simulations ont été réalisées pour les conditions expérimentales du diagramme de la fig. $10 \mathrm{~b}$, pour le réseau $\left[\mathrm{Ca}_{2} \mathrm{CoO}_{3}\right]$ seul (fig. 10c), pour le réseau 

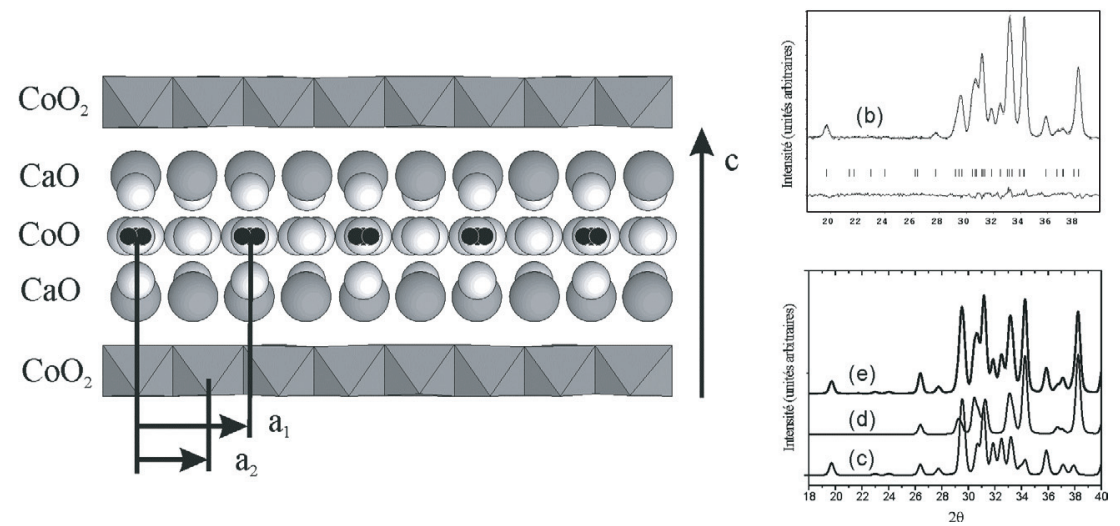

Figure 10. $\left[\mathrm{Ca}_{2} \mathrm{CoO}_{3}\right]\left[\mathrm{CoO}_{2}\right]_{1.62}$ : (a) représentation schématique de la structure et du désordre de la couche centrale $\mathrm{CoO}$ (b) affinement à partir du diagramme de diffraction des neutrons ; (c), (d) diagrammes de diffraction simulés pour le réseau $\left[\mathrm{Ca}_{2} \mathrm{CoO}_{3}\right]$ seul, pour le réseau $\left[\mathrm{CoO}_{2}\right]$ seul, et (e) diagramme somme de (c) et (d).

$\left[\mathrm{CoO}_{2}\right]$ seul (Fig. 10d) et on a représenté en Fig. 10e la somme des deux simulations précédentes. On remarque bien sur les figures $10 \mathrm{c}$ et $10 \mathrm{~d}$ des réflexions communes $(0 \mathrm{kl} / 0)$, se positionnant aux mêmes angles de diffraction, ainsi que des réflexions spécifiques à chacun des réseaux ( $h \mathrm{kl} 0 \mathrm{ou} 0 \mathrm{klm})$, qui vont dépendre spécifiquement de chacune des métriques correspondantes. Enfin, il est clair aussi que le diagramme réel n'est pas la somme de deux diagrammes indépendants, mais que la cohérence globale de la structure composite doit être prise en compte pour la modélisation de l'ensemble de la structure, d'où la nécessité de travailler dans le formalisme adéquat de superespace, ainsi qu'avec tous les outils de symétrie associés qui ne sont pas développés ici.

Ces structures lamellaires d'oxydes de cobalt présentent différents types d'arrangement en fonction de la nature des couches, mais gardent systématiquement le même principe d'empilement. Une analyse systématique de différentes structures de ce type a également montré la similarité des couches $\mathrm{CoO}_{2}$ du deuxième sous-système, qui tend à montrer la rigidité ainsi que la stabilité spécifique de ce type de couches, qui est responsable des propriétés thermoélectriques de ces structures. Certaines structures révèlent un degré de complexité supplémentaire en associant une modulation commensurable ou incommensurable, indépendante du caractère composite, et nécessitant pour la résolution structurale un formalisme à 5 dimensions [18].

\section{CONCLUSION}

Il apparaît ainsi que la cristallographie a dû prendre en compte depuis maintenant une trentaine d'années, de nouvelles structures caractérisées par un ordre à grande distance et la perte d'une périodicité dans l'espace physique. Pour les exemples correspondants qui concernent les quasicristaux, les structures modulées et les structures composites, l'ordre à grande distance a pu à nouveau être associé à une «superstructure» périodique, mais dans un espace de dimension supérieure, selon une démarche de section-projection. Il convient alors de travailler avec des outils cristallographiques généralisés, permettant d'inclure des modélisations atomiques spécifiques, qui ne sont plus ponctuelles et dont la forme peut être le résultat de l'affinement. Il faut aussi insister sur la nécessité de travailler dans une symétrie compatible avec ces nouveaux objets et ainsi de pouvoir associer à une telle structure un supergroupe d'espace [19]. Il est aussi important de noter que ce qui pouvait apparaître comme une découverte anecdotique à ses débuts, se révèle en fait beaucoup plus fréquent qu'on ne pouvait l'imaginer alors, ce qui justifie a posteriori tout l'effort qui a pu être fait sur le plan théorique, cristallographique et logiciel pour répondre à cette demande. 


\section{Remerciements}

Je tiens à remercier Marc de Boissieu pour les renseignements et les images fournies sur les quasicristaux, ainsi que N. Jakubowicz, S. Lambert, N. Guiblin, O. Pérez et H. Rousselière pour les études concernant les structures modulées et composites.

\section{Références}

[1] Lifshitz R., arXiv:cond-mat/0701029v1 (2007).

[2] Janner A., Acta Cryst. B 51, 386 (1995).

[3] Jannsen T., Janner A., Looijenga-Vos A. and de Wolff P. M., International Tables for Crystallography, vol. C, edited by A. J. C. Wilson (Dordrecht: Kluwer Academic Publishers), 797 (1992).

[4] Yamamoto A., Acta Cryst. A 52, 509 (1996).

[5] Duneau M. and Katz A., Phys. Rev. Lett. 54, 2688 (1985); Katz, A. and Duneau M., J. Phys. France 47, 181 (1986).

[6] Lambert S., Leligny H. and Grebille D., J. Solid State Chem. 160, 322 (2001).

[7] Yamamoto A. and Takakura H., Phys. Rev. B 68, 094201 (2003).

[8] Weber S. and Yamamoto A., Acta Cryst. A 54, 997 (1998).

[9] Guiblin N., Ph.D. Thesis, Université de Caen-Basse Normandie (2004).

[10] Petricek V. and Dusek M., JANA2000, Institut de Physique, Prague, République tchèque.

[11] Daoud-Aladine A., Rodriguez-carvajal J., Pinsard-Gaudart L., Fernandez-Diaz M. T. and Revcolevschi A., Phys. Rev. Lett. B 89, 097205 (2002).

[12] Cox D. E., Radaelli P. G., Marezio M. and Cheong S. W., Phys. Rev. B 57, 3305 (1998).

[13] Jakubowicz N., Grebille D., Leligny H. and Evain M., J. Phys.: Cond. Matter 11, 3997 (1999).

[14] Etrillard J., Bourges Ph. and Lin C. T., Phys. Rev. B 62, 150 (2000).

[15] Grebille D. and Leligny H., Pérez O., Phys. Rev. B 64, 106501 (2001).

[16] Dusek M., Chapuis G., Schobinger-Papamantellos P., Wilkinson C., Petricek V., Tung L. D. and Buschow K. H. J., Acta Cryst. B 56, 959 (2000).

[17] Grebille D., Lambert S., Bourée F. and Petricek V., J. Appl. Cryst. 37, 823 (2004).

[18] Leligny H., Grebille D., Pérez O., Masset A. C., Hervieu M. and Raveau B., Acta Cryst. B 56, 173 (2000).

[19] Brown H., Bülow R., Neubûser J. and Wondratschek H., Crystallographic Groups of FourDimensional Space (ed) (New York, John Wiley, 1978); Janssen T., Birman J. L, Denoyer F., Koptsik V. A., Verger-Gaugry J. L., Weigel D., Yamamoto A., Abrahams S. C. and Kopsky V., Acta Cryst. A 58, 605 (2002); Janner A., Janssen T., Acta Cryst. A 36, 399 (1980); Janner A. and Janssen T., Acta Cryst. A 36, 408 (1980). 